\title{
Reactivity of Triarylphosphine Peroxyl Radical Cations \\ Generated through the Reaction of Triarylphosphine Radical
}

\section{Cations with Oxygen}

\author{
Sachiko Tojo ${ }^{1}$, Shinro Yasui ${ }^{2}$, Mamoru Fujitsuka ${ }^{1}$, and Tetsuro Majima ${ }^{1}$ * \\ ${ }^{1}$ The Institute of Scientific and Industrial Research (SANKEN), Osaka University \\ Mihogaoka 8-1, Ibaraki, Osaka 567-0047, Japan \\ ${ }^{2}$ Laboratory of Biology and Chemistry, Tezukayama University, Gakuen Minami 3, \\ Nara 631-8585, Japan \\ E-mail: majima@sanken.osaka-u.ac.jp
}

\section{Table of Contents}

Figure S1. Transient absorption spectra observed during the pulse radiolyses of $\mathbf{A r}_{3} \mathbf{P}(10 \mathrm{mM})$ in Ar-saturated PhCN. page S2-S3

Figure S2. Transient absorption spectra observed during the pulse radiolyses of $\mathbf{1 d}$ and $\mathbf{1 h}(10 \mathrm{mM})$ in $\mathrm{O}_{2}$-saturated $\mathrm{PhCN}$. page S4

Table S1. Cartesian Coordinates of $\mathbf{1 f}^{\bullet+}$ -page S5 
A

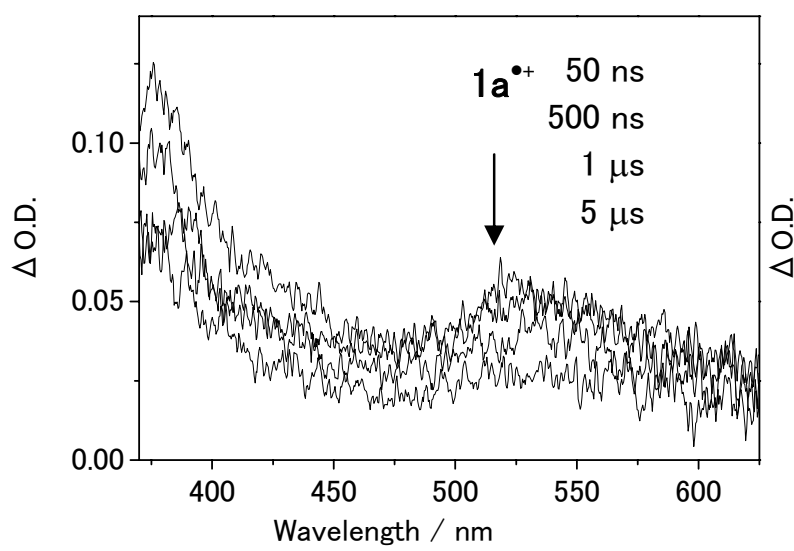

B

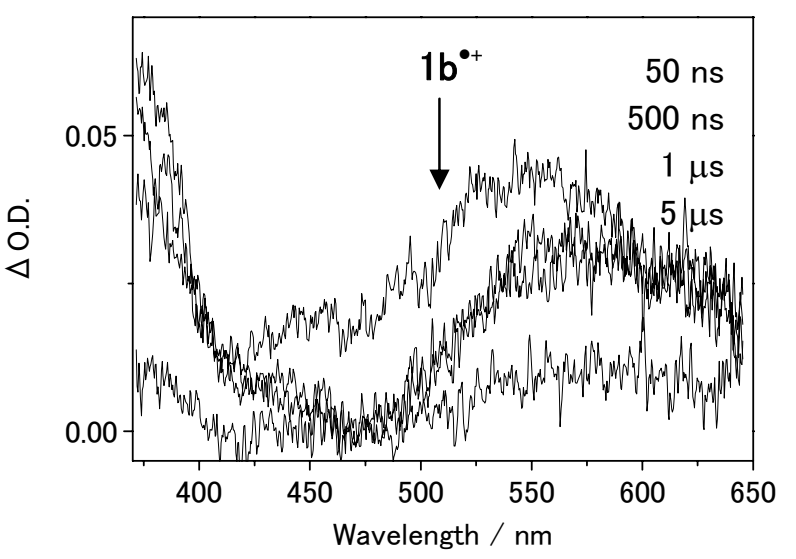

D
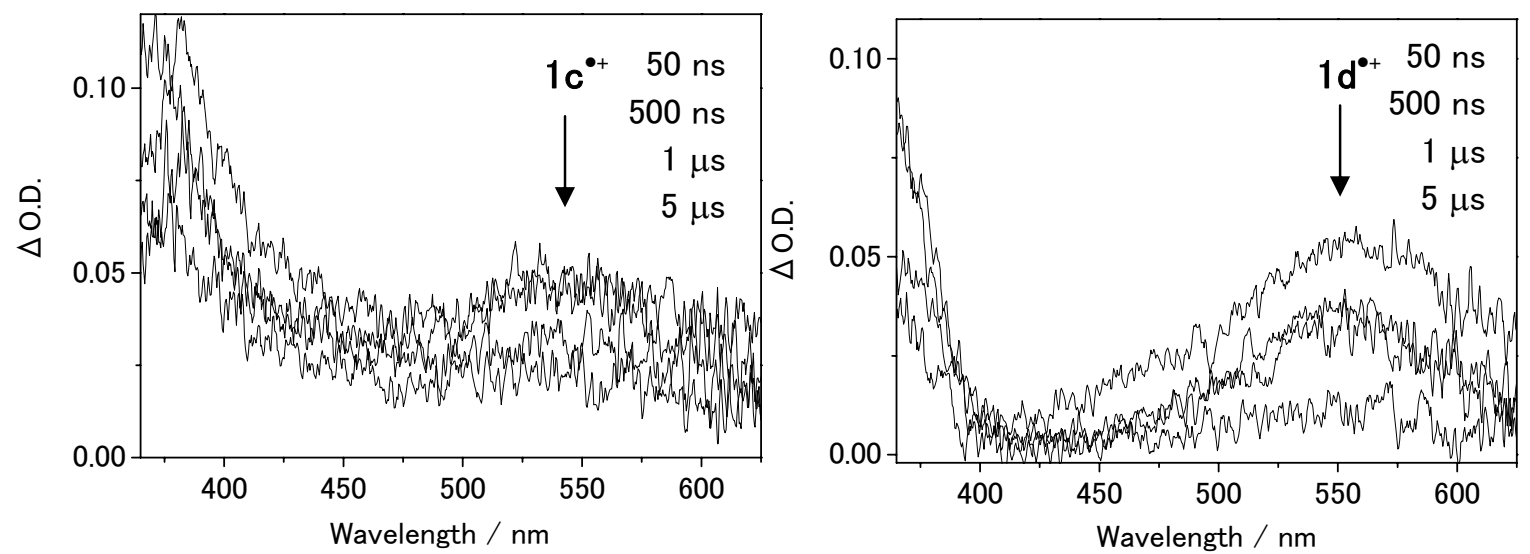

E

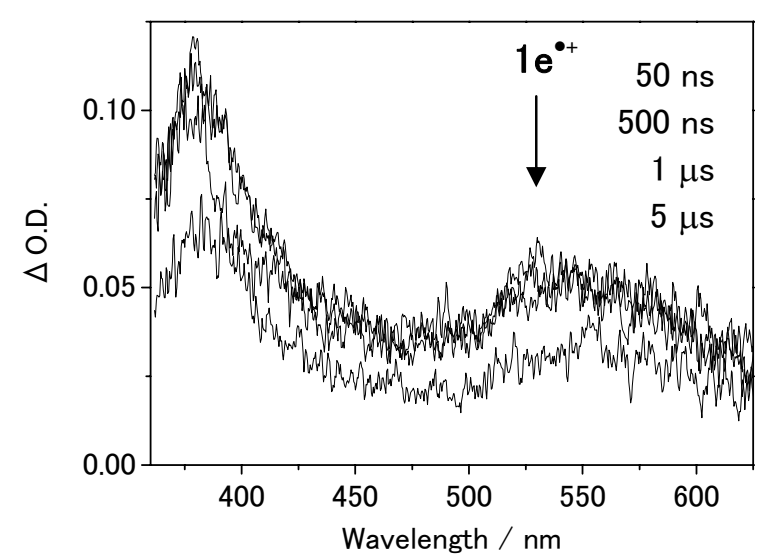

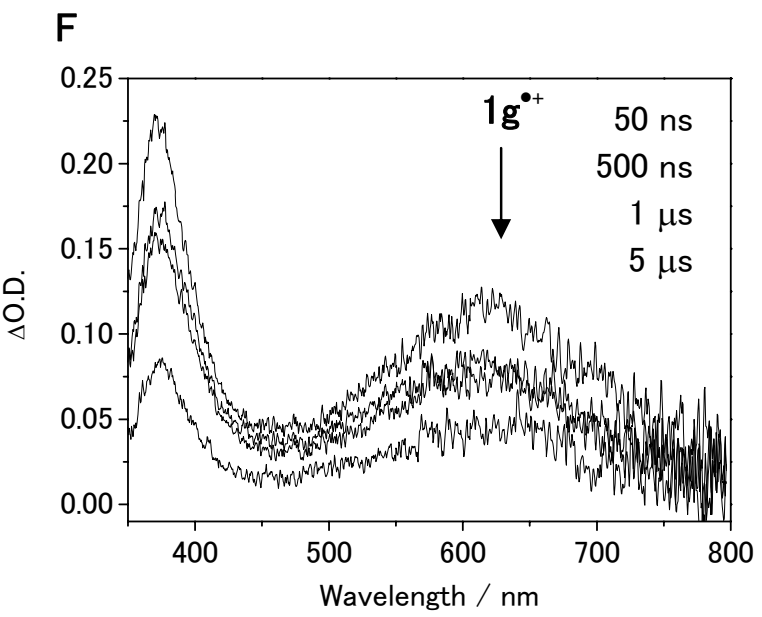


G

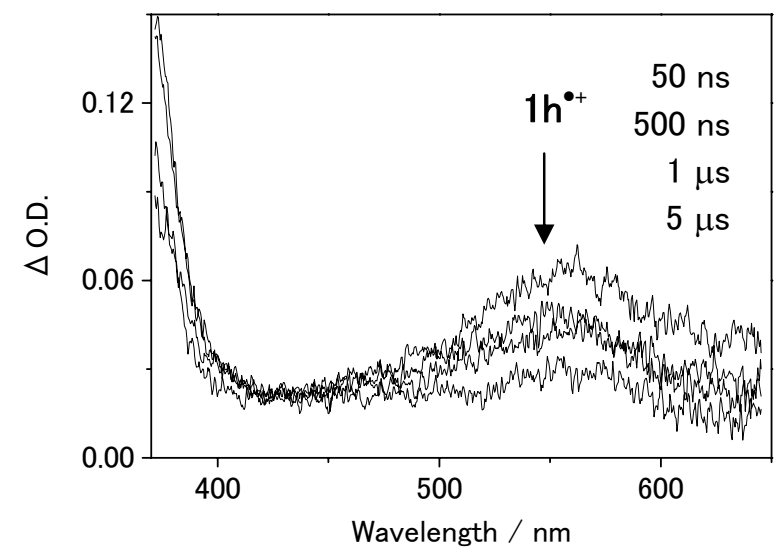

I

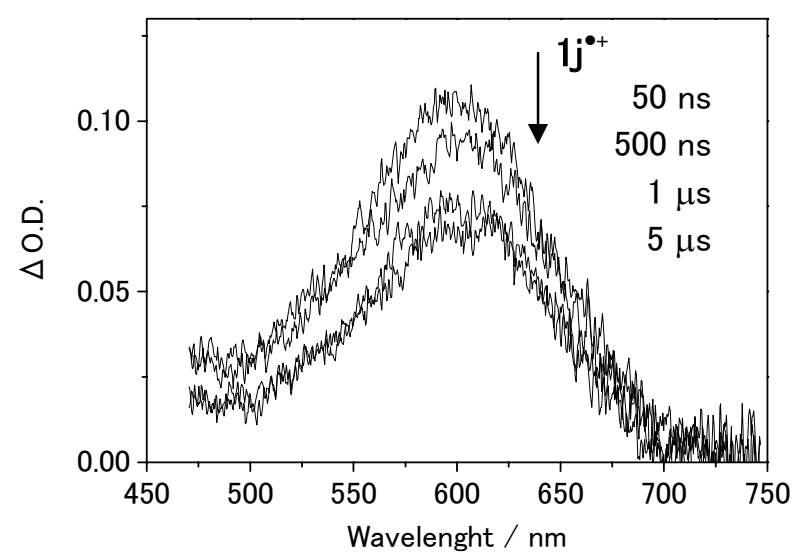

$\mathrm{K}$

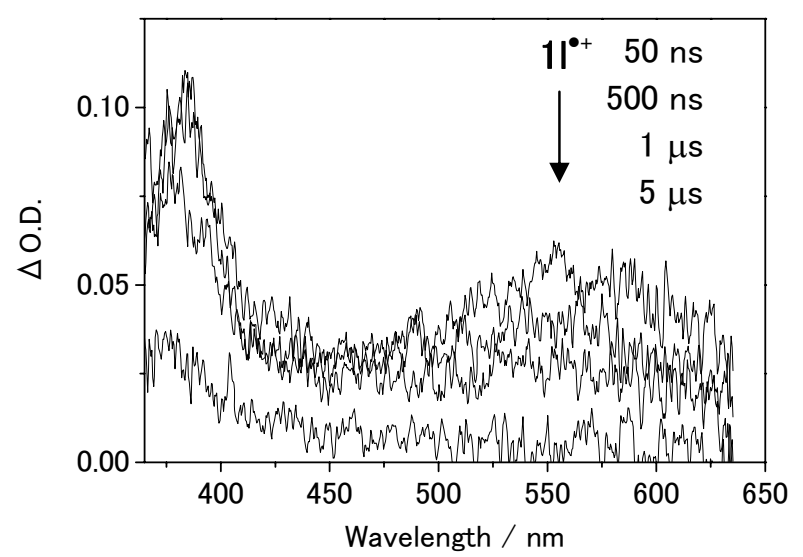

$\mathrm{H}$

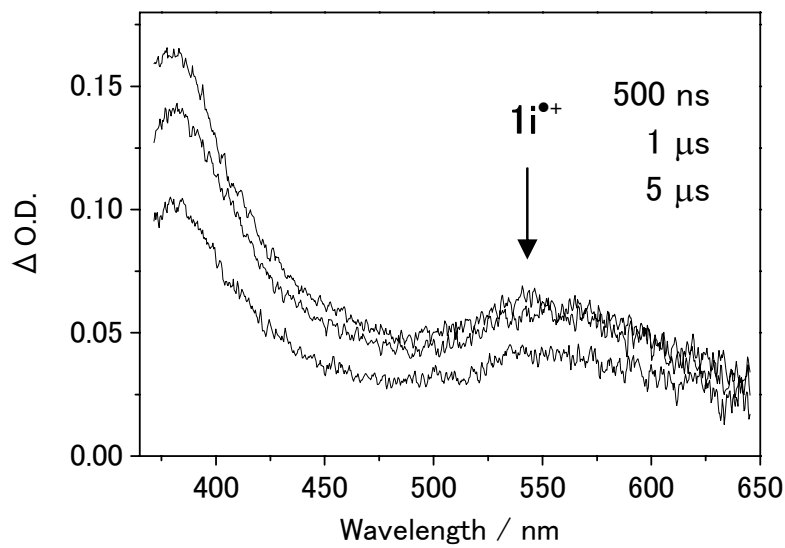

J

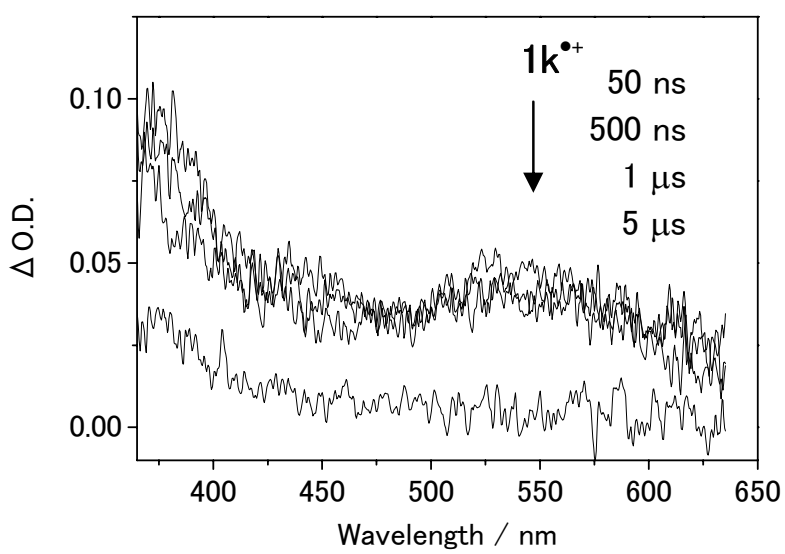

Figure S1. Transient absorption spectra observed at 50 ns, 500 ns, $1 \mu \mathrm{s}$, and $5 \mu$ ss after an electron pulse during the pulse radiolysis of $\mathbf{1}(10 \mathrm{mM}$ ) in Ar-saturated PhCN. (A) 1a, (B)1b, (C)1c, (D)1d, (E)1e, (F)1g, (G)1h, (H)1i, (J)1k, and (K)1l. 
A

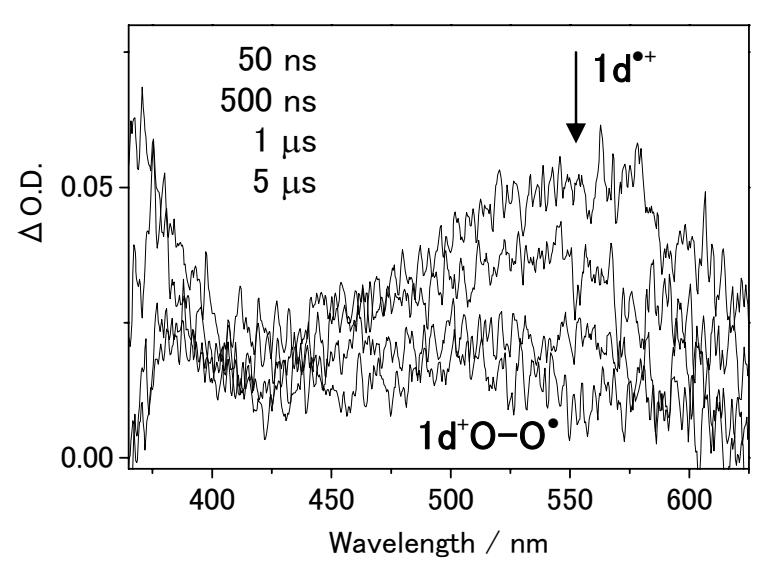

B

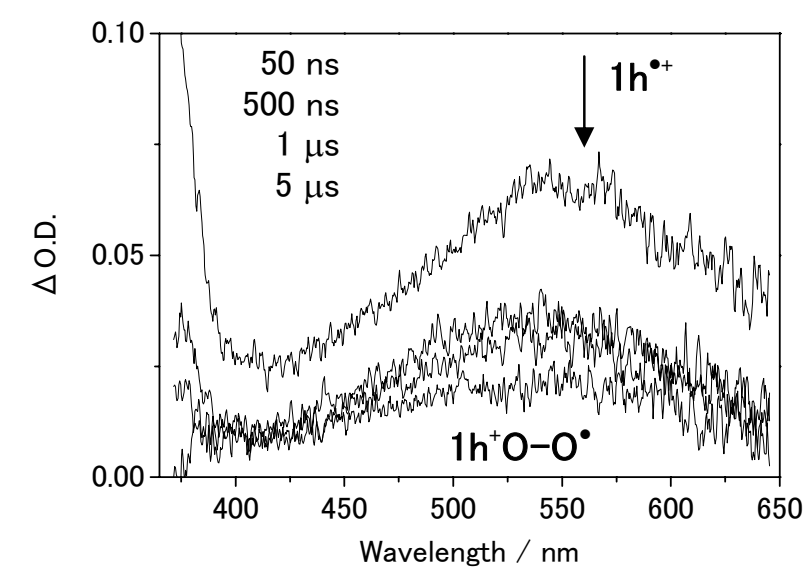

Figure S2. Transient absorption spectra observed at 50 ns, 500 ns, $1 \mu$ s, and $5 \mu$ ss after an electron pulse during the pulse radiolysis of $\mathbf{1}(10 \mathrm{mM})$ in $\mathrm{O}_{2}$-saturated PhCN. (A) 1d, (B)1 $\mathrm{h}$. 
Table S1. Cartesian Coordinates of $1 \mathbf{f}^{\mathbf{0}^{+}}$. (Total energy: -1153.637550 Hartree). ${ }^{\mathrm{a}}$

\begin{tabular}{|c|c|c|c|}
\hline & \multicolumn{3}{|c|}{ Coordinates (Angstroms) } \\
\hline & $\mathrm{X}$ & Y & Z \\
\hline $\mathrm{P}$ & 0.00071 & -0.00045 & -0.79163 \\
\hline C & -0.44245 & 1.67126 & -0.33592 \\
\hline $\mathrm{C}$ & -1.22648 & -1.21973 & -0.33823 \\
\hline $\mathrm{C}$ & 1.6693 & -0.45334 & -0.33461 \\
\hline C & -2.53122 & -1.09675 & -0.86605 \\
\hline C & -3.49693 & -2.04524 & -0.56029 \\
\hline C & -3.19953 & -3.15005 & 0.25745 \\
\hline C & -1.8925 & -3.27345 & 0.76005 \\
\hline C & -0.91281 & -2.33335 & 0.46836 \\
\hline C & 2.47275 & 0.37215 & 0.47941 \\
\hline C & 3.77373 & -0.00955 & 0.77996 \\
\hline C & 4.32322 & -1.20094 & 0.275 \\
\hline C & 3.51737 & -2.01079 & -0.54498 \\
\hline C & 2.21612 & -1.64536 & -0.85974 \\
\hline C & -1.55862 & 1.95504 & 0.4783 \\
\hline $\mathrm{C}$ & -1.87758 & 3.27289 & 0.77884 \\
\hline C & -1.12013 & 4.34389 & 0.27336 \\
\hline $\mathrm{C}$ & -0.01624 & 4.05011 & -0.54677 \\
\hline C & 0.31712 & 2.74022 & -0.86118 \\
\hline C & -1.49785 & 5.76897 & 0.5778 \\
\hline $\mathrm{C}$ & -4.25854 & -4.16293 & 0.6017 \\
\hline C & 5.74621 & -1.58556 & 0.58028 \\
\hline $\mathrm{H}$ & -2.78326 & -0.26515 & -1.51834 \\
\hline $\mathrm{H}$ & -4.49684 & -1.93862 & -0.97172 \\
\hline $\mathrm{H}$ & -1.64315 & -4.11928 & 1.39504 \\
\hline $\mathrm{H}$ & 0.08527 & -2.45024 & 0.87739 \\
\hline $\mathrm{H}$ & 2.07176 & 1.29142 & 0.8933 \\
\hline $\mathrm{H}$ & 4.37565 & 0.62309 & 1.42669 \\
\hline $\mathrm{H}$ & 3.9233 & -2.93428 & -0.94846 \\
\hline $\mathrm{H}$ & 1.62293 & -2.28072 & -1.51168 \\
\hline $\mathrm{H}$ & -2.15453 & 1.14847 & 0.89234 \\
\hline $\mathrm{H}$ & -2.7261 & 3.47855 & 1.42575 \\
\hline $\mathrm{H}$ & 0.581 & 4.86298 & -0.95052 \\
\hline $\mathrm{H}$ & 1.16416 & 2.54353 & -1.51265 \\
\hline $\mathrm{H}$ & -2.19578 & 6.15016 & -0.17965 \\
\hline $\mathrm{H}$ & -0.62347 & 6.42654 & 0.57382 \\
\hline $\mathrm{H}$ & -1.99348 & 5.85494 & 1.54933 \\
\hline $\mathrm{H}$ & -4.78906 & -3.86679 & 1.51664 \\
\hline $\mathrm{H}$ & -3.82604 & -5.15145 & 0.78229 \\
\hline $\mathrm{H}$ & -5.00511 & -4.24987 & -0.19326 \\
\hline $\mathrm{H}$ & 6.06638 & -1.20254 & 1.5538 \\
\hline $\mathrm{H}$ & 6.42579 & -1.16754 & -0.17443 \\
\hline $\mathrm{H}$ & 5.88032 & -2.67129 & 0.57233 \\
\hline
\end{tabular}

${ }^{\mathrm{a}}$ Calculated at UB3LYP/6-31Gd level. 